\title{
Zeeman and Orbital Effects of an in-Plane Magnetic Field in Cuprate Superconductors
}

\author{
Kun Yang \\ National High Magnetic Field Laboratory and Department of Physics, Florida State University, Tallahassee, Florida 32310 \\ S. L. Sondhi \\ Department of Physics, Princeton University, Princeton, New Jersey 08544
}

(October 28, 2018)

\begin{abstract}
We discuss the effects of a magnetic field applied parallel to the $\mathrm{Cu}-\mathrm{O}(a b)$ plane of the high $T_{c}$ cuprate superconductors. After briefly reviewing the Zeeman effect of the field, we study the orbital effects, using the Lawrence-Doniach model for layered superconductors as a guide to the physics. We argue that the orbital effect is qualitatively different for in-plane and inter-layer mechanisms for superconductivity. In the case of in-plane mechanisms, interlayer couplings may be modeled as a weak interlayer Josephson coupling, whose effects disappear as $H \rightarrow \infty$; in this case Zeeman dominates the effect of the field. In contrast, in the inter-layer mechanism the Josephson coupling is the driving force of superconductivity, and we argue that the in-plane field suppresses superconductivity and provides an upper bound for $H_{c 2}$ which we estimate very crudely.
\end{abstract}

One of the most important milestones in the study of the cuprate superconductors is the identification of the predominantly $d_{x^{2}-y^{2}}$ symmetry of their pairing order parameters. This discovery has motivated a tremendous amount of theoretical work on the physical properties of superconductors with unconventional pairing symmetry.

Among them, it was recently pointed out that the response of a two-dimensional (2D) $d_{x^{2}-y^{2}}$ superconductor to the Zeeman coupling between the spins of the electrons and an external magnetic field is much stronger than that in an $s$-wave superconductor. This is so, for unlike the fully gapped $s$-wave case, in a $d_{x^{2}-y^{2}}$ superconductor there exists nodal points in the order parameter and therefore gapless quasiparticle excitations. Consequently, an arbitrarily weak Zeeman field generates a finite density of spin-polarized quasiparticles in the ground stated. It was also shown that the Zeeman field enhances the low- $T$ specific heat, thermal conductivity, and tunneling dep ity of states, while suppressing the superfluid density 1 . At strong fields, the system supports a Fulde-Ferrell-Larkin-Ovchinnikov type superconducting state.3. Eventually, a sufficiently strong Zeeman field suppresses superconductivity completely, leading to a "Pauli limit" on the upper critical field.

The results mentioned above are expected to be particularly relevant to the cuprate superconductors when the external field is oriented parallel to the $\mathrm{Cu}-\mathrm{O}(a b)$ planes (for possible caveats on the influence of disorder see [ [1]), as the cuprates are quasi-2D systems with electrons predominantly moving in the $\mathrm{Cu}-\mathrm{O}$ planes. In a truly $2 \mathrm{D}$ system, the orbital motions of the electrons would not sense the existence of a parallel magnetic field, and the Zeeman effect would be the only effect of the field. Experimentally, there is some evidence 5 suggesting that the upper critical field for the parallel orientation is indeed Pauli limited in YBCO at low temperatures.

However for smaller fields there do exist orbital effects, even when the field is parallel to the planes, due to the presence of inter-plane couplings. It is the purpose of this paper to report results of studies of such orbital effects, and to compare them with the Zeeman effect in field ranges that are relevant to current experiments. We will argue that the nature and importance of the orbital effects depend sensitively on whether superconductivity is driven by in-plane correlations or inter-plane hopping. Thus their study may provide a way to distinguish between in-plane and inter-plane mechanisms for superconductivity in the cuprates.

The orbital effects of the parallel field may be summarized qualitatively as the following. The field generates screening currents both within and across the planes. The current within the plane generates a "Doppler shift" of the quasiparticle spectrumb, and therefore a finite density of states (DOS) for the quasiparticles. This currentinduced DOS is proportional to $\sqrt{H}$ for $H \| d$, and thus dominates the Zeeman effect (which gives rise to a quasiparticle DOS that is proportional to $H$ ) at low $H$. We show below that the situation is quite different for an inplane field $(H \perp \hat{c})$. Also the inter-plane Josephson current suppresses the inter-plane Josephson coupling energy. Thus, depending on how crucial the Josephson coupling energy is to superconductivity itself, the response of the system to the in-plane field can be very different.

We model the cuprates by the Lawrence-Doniach model 1 that is appropriate for layered superconductors:

$$
\begin{aligned}
& F=\sum_{n} \int d x d y\left\{\alpha\left|\psi_{n}\right|^{2}+\frac{\beta}{2}\left|\psi_{n}\right|^{4}\right. \\
& \left.+\frac{\hbar^{2}}{2 m_{a b}}\left|\left(\nabla-\frac{2 i e}{\hbar c} \mathbf{A}_{n}\right) \psi_{n}\right|^{2}+t\left|\psi_{n+1}-\psi_{n}\right|^{2}\right\} .
\end{aligned}
$$

Here $n$ is the layer index, $\alpha \propto T-T_{c}$, and other parameters may be assumed to be $T$ independent. For $T$ very close to $T_{c}$, the coherence length becomes very large, so 
that continuum approximation can be used along the $c$ direction, and (11) reduces to the usual Ginsburg-Landau theory with anisotropic mass, and the mass along the $c$ direction is $m_{c}=\frac{\hbar^{2}}{2 s^{2} t}$ where $s$ is the spacing between layers. The parameter $\gamma^{2}=m_{c} / m_{a b}$ is a measure of the degree of anisotropy of the system; for examples, $\gamma^{2} \approx 50$ for YBCO, and $\gamma^{2} \approx 20000$ for BSCCO 10 .

We are primarily interested in the low $T$ regime, where the layered structure is important; more precisely we are interested in the case $T<T^{*}$, the latter being the temperature at which the orbital $H_{c 2}$ diverges. At such temperatures we distinguish two regimes: a low field regime in which the current flowing in the layers increases with $H$ on account of increasing vortex density, and a high field regime in which the increasing density is counteracted by the cancelation between neighboring vortex patterns. The former has also been discussed by Volovik the answer at high fields. We assume the external field $\mathbf{H}=H(1,0,0)$ is along the $\hat{a}$ direction. It is known that $H_{c 1}$ is very low $(<100$ Gauss $)$ for a parallel field, and for fields of order $H \sim 1 T$ (which is the range of interest in the present paper and typical for experimental studies), the field basically penetrates the system uniformly and the screening effect is negligible. (This justifies neglecting the field energy term in (1i)). Thus we can assume the field inside the superconductor is the same as that of the external field and uniform. We use the gauge $\mathbf{A}_{n}=n s H(0,1,0)$.

We need to solve the equation for $\psi_{n}$ :

$$
\begin{aligned}
\frac{\delta F}{\delta \psi_{n}^{*}} & =\alpha \psi_{n}+\beta\left|\psi_{n}\right|^{2} \psi_{n}-\frac{\hbar^{2}}{2 m_{a b}}\left(\nabla-\frac{2 i e}{\hbar c} \mathbf{A}_{n}\right)^{2} \psi_{n} \\
& -t\left(\psi_{n-1}+\psi_{n+1}\right)=0 .
\end{aligned}
$$

For in-plane mechanisms, the Josephson coupling $t$ is weak and secondary to superconductivity. To find the solution to (11), we first set $t=0$, and then treat $t$ as a weak perturbation. For $t=0$, all the layers decouple, and we obtain

$$
\psi_{n}(x, y)=\sqrt{-\alpha / \beta} e^{i\left(\phi_{n}+k_{n} y\right)},
$$

where $k_{n}=\frac{2 e n s H}{\hbar c}$ and $\phi_{n}$ are arbitrary phases. Upon introducing $t$ these solutions spread to more than one layer and llock phases, leading to the unique, leading order solution 11

$$
\psi_{n}(x, y)=\sqrt{\frac{-\alpha}{\beta}} e^{i k_{n} y}\left[\eta_{n}+\frac{2 i \eta_{n+1}}{\gamma^{2}}\left(\frac{\ell}{s}\right)^{4} \sin (\Delta k y)\right],
$$

where $\Delta k=2 e s H / \hbar c, \ell=\sqrt{\hbar c / 2 e H}$ is the magnetic length for Cooper pairs, $\eta_{n}=1$ for $n=4 j$ or $4 j+1$, and -1 otherwise ( $j$ is an integer). In order for the perturbative approach to be valid, we need to have

$$
\frac{1}{\gamma^{2}}\left(\frac{\ell}{s}\right)^{4} \ll 1
$$

which is always valid in the strong field limit.

The typical in-plane superfluid velocity is thus

$$
v_{s} \approx \frac{\hbar \Delta k}{\gamma^{2} m_{a b}}\left(\frac{\ell}{s}\right)^{4},
$$

and the Doppler shift energy is

$$
E_{D} \sim \hbar v_{s} k_{F}=\frac{\epsilon_{F}}{\gamma^{2}} \frac{\ell^{2}}{k_{F} s^{3}} \propto 1 / H
$$

Since the Zeeman energy $E_{Z}=\mu_{B} H \propto H$, it always dominates the Doppler shift at sufficiently strong field. At field $H=10 T$, using $\epsilon_{F} \sim 2 e V, k_{F} \sim 1 \AA^{-1}$ and $s \sim 10 \AA$, we obtain for BSCCO (in which (5) is satisfied) $E_{D} / E_{Z} \sim 0.4$, indicating Zeeman effect starts to dominate the orbital effect in this field range in BSCCO. (5) is not satisfied in YBCO at this field due to less anisotropy; but using the above results anyway (which results in an overestimate of the in-plane current) we find $E_{D} / E_{Z} \sim 150$, suggesting that the orbital effect will dominate.

In the above discussion we have assumed that the interlayer Josephson couplings are secondary to superconductivity in cuprates, and may be treated as weak perturbations. This is appropriate for in-plane mechanisms for superconductivity in cuprates However it was proposed by Anderson and coworkers 1213 that the interlayer Josephson coupling (or pair-hopping) may actually be the driving force behind superconductivity in cuprates. In this case, the interlayer Josephson coupling can no longer be treated as a weak perturbation as above, and we expect an in-plane magnetic field to have a much more dramatic orbital effect. This is because the in-plane field tends to suppress interlayer phase coherence, and therefore Josephson energy and superconductivity in this case. Among other things, the orbital effect of the field provides a mechanism for the upper critical field $H_{c 2}$. While for in-plane mechanisms, the field simply decouples the layers, and $H_{c 2}$ can only come from the Zepman effectll.

In the inter-layer pair hopping mode 12 . 23 , the multilayer structure of the cuprates is important. In particular, the Josephson coupling among layers within the same unit cell might be much stronger than between different cells, thus the large anisotropy could largely reflect the weakness of the inter-unit cell Josephson coupling only. For simplicity, we study in the following in a single bilayer (as in Ref. 13), and neglect the coupling between this pair of layers and the rest of the system. A proper treatment of even this problem requires a microscopic theory that does not really exist. So here we will content ourselves with a speculative illustration of what such a treatment might produce. To this end we again use the Lawrence-Doniach model, in a slightly different form and with a crucial reinterpretation of the parameters:

$$
F=\int d x d y\left\{\alpha^{\prime}\left(\left|\psi_{1}\right|^{2}+\left|\psi_{2}\right|^{2}\right)+\frac{\beta}{2}\left(\left|\psi_{1}\right|^{4}+\left|\psi_{2}\right|^{4}\right)\right.
$$




$$
\begin{aligned}
& +\frac{\hbar^{2}}{2 m_{a b}}\left(\left.\left|\nabla \psi_{1}\right|^{2}|+| \nabla \psi_{2}\right|^{2} \mid\right) \\
& \left.-t\left(\psi_{1}^{*} \psi_{2} e^{\frac{2 i e H s x}{\hbar c}}+\psi_{2}^{*} \psi_{1} e^{-\frac{2 i e H s x}{\hbar c}}\right)\right\} .
\end{aligned}
$$

Here $\alpha^{\prime}=\alpha+t$. We have used a different gauge: For a parallel field $\mathbf{H}=H \hat{y}$, we choose $\mathbf{A}=H x \hat{z}$, so the $\mathbf{A}$ affects the phase of the interlayer Josephson coupling, but not in-plane kinetic energy. The physics, of course, is unaffected by the choice of gauge. The reason to isolate the last term out is that this term describes interlayer coupling only; while all the in-plane properties are described by other terms.

For in-plane mechanisms, each individual layer is superconducting (at low $T$ ) without interlayer coupling. Thus we must have $\alpha^{\prime}<0$, which means the reason that a superconducting order parameter $\psi$ is developed is because of the lowering of in-plane free energy. Without a parallel field, the free energy can be further lowered by having the same phase for $\psi_{1}$ and $\psi_{2}$ (and uniform throughout the system so that there is no in-plane kinetic energy cost), so that the systems gains Josephson coupling energy as well.

In pair hopping mechanism, however, the individual layers are not superconducting without interlayer Josephson coupling. This means $\alpha^{\prime}>0$ ! Instead the system becomes superconducting at $H=0$ by developing a nonzero $\psi$ in both layers, and making them phase coherent $\left(\psi_{1}=\psi_{2}\right)$, thereby gaining Josephson coupling energy. Thus as long as $t>\alpha^{\prime}$, the system is in the superconducting phase, despite $\alpha^{\prime}>0$.

As long as $H=0$, the ground state of $(8)$ is the same: $\psi_{1}=\psi_{2}=\sqrt{\left(t-\alpha^{\prime}\right) / \beta}$, and there is no obvious distinction between these two cases; the system is phase coherent both within each individual layer and between the two layers. The situation is very different when $H \neq 0$ and is sufficiently large. This is because for a loop that encloses a finite amount of flux, one must accumulate a finite amount of gauge-invariant phase difference proportional to the flux (see Fig. 1a). It is therefore no-longer possible to maintain in-plane and interlayer phase coherence simultaneously 4 .

For $\alpha^{\prime}<0$ (in-plane mechanism), the situation is basically the same as a Josephson junction in a magnetic field10; we have $\psi_{j}=e^{i \phi_{j}} \sqrt{-\alpha^{\prime} / \beta}$, with no correlation between $\phi_{1}$ and $\phi_{2}$. The leading order correction to this is proportional to $t / H$. The field induces an oscillatory Josephson current between the layers: $j_{z} \approx\left(-\alpha^{\prime} t / \beta\right) \sin \left(\frac{2 e s H x}{\hbar c}+\phi_{1}-\phi_{2}\right)$, while the in-plane current goes to zero as $1 / H$ at large $H$ (see Fig. 1b). The Josephson coupling term in (8) averages to zero in this solution. In short, the system gives up interlayer coherence (as manifested by the loss of Josephson coupling energy and appearance of Josephson current), in order to maintain in-plane coherence (therefore no in-plane current and kinetic energy).

For $\alpha^{\prime}>0$ (interlayer mechanism), on the other hand, the system will do everything possible to maintain inter- layer coherence, as the Josephson energy is the origin of superconductivity. Thus the solution in this case is

$$
\psi_{1,2}=\sqrt{\left(t+\alpha^{\prime}-\frac{(e s H)^{2}}{2 m_{a b} c^{2}}\right) / \beta} e^{ \pm i e s H x / \hbar c} .
$$

In this solution the interlayer coherence is maintained and there in no interlayer Josephson current induced by the field. As a price, the system loses in-plane coherence, and there is diamagnetic current $(\propto H)$ and in-plane kinetic energy loss $\left(\propto H^{2}\right)$ (see Fig. 1c). Clearly, as $H$ increases, at some point the loss of in-plane kinetic energy will overwhelm the gain of Josephson energy, and the system will cease to superconduct. This is the orbital upper critical field $H_{c 2}^{o}$ (to be distinguished from the Zeeman upper critical field $H_{c 2}^{Z}$ ), as determined by the point where $\psi$ vanishes in (9). For in-plane mechanisms, $H_{c 2}^{o} \rightarrow \infty$. If we assume that interlayer mechanism is responsible for superconductivity in cuprates, a crude estimate yields in BSCCO2212 $\mathrm{H}_{c 2}^{o} \sim 200 T$ at $T=0$. Unfortunately this is comparable to $H_{c 2}^{Z}$ ㄴ. 3 . However, in principle, the Zeeman effect can be suppressed by introducing spin-orbital scatterers to the system which could enable the two to be distinguished.

The qualitative difference in the orbital response to a parallel magnetic field can provide a way to experimentally distinguish between in-plane and interlayer mechanisms for cuprate superconductivity. We note the interpretation of previous experimental attempts to distinguish between the two based on $c$-axis penetration depth is controversial15.

This work was supported by NSF DMR-9971541 and the Sloan Foundation (KY), and NSF DMR-9632690, and Sloan and Packard Foundations (SLS).

${ }^{1}$ K. Yang and S. L. Sondhi, Phys. Rev. B 57, 8566 (1998).

${ }^{2}$ H. Won, H. Jang and K. Maki, cond-mat/9901252 (1999).

${ }^{3}$ K. Maki and H. Won, Czech. J. Phys. 46, 1035 (1996).

${ }^{4}$ For discussions of influence of impurity and spin-orbit scatterings on the Zeeman effect in $d$-wave superconductors, see C. Grimaldi, cond-mat/9905262 and cond-mat/9907004.

${ }^{5}$ J. L. O'Brein et al., cond-mat/9901341.

${ }^{6}$ S. K. Yip and J. A. Sauls, Phys. Rev. Lett. 69, 2264 (1992).

${ }^{7}$ G. E. Volovik, JETP Lett. 58, 469 (1993).

${ }^{8}$ W. E. Lawrence and S. Doniach, in Proceedings of the 12th International Conference on Low Temperature Physics, Edited by E. Kanda (Academic, Kyoto, 1971), p. 361.

${ }^{9}$ In the following model it is assumed that each unit cell along the $\hat{c}$ direction contains one layer, and all layers are equivalent. Many cuprates, however, have multiple layers in a unit cell. Such multi-layered structure is not very important for in-plane mechanisms for superconductivity, but jisvery important for the interlayer pair hopping mechanism 12. See discussions later. 
${ }^{10}$ M. Tinkham, Introduction to Superconductivity, second edition, McGraw-Hill, New York (1996).

${ }^{11}$ Our results below differ from a previous study (S. Theodorakis, Phys. Rev. B 42, 10172 (1990)) which appears to use a similar approach.

${ }^{12} \mathrm{P}$. W. Anderson, The Theory of Superconductivity in the High-T Cuprates, Princeton Univ. Press, Princeton (1997).

13 S. Chakravarty, A. Sudbø, P. W. Anderson, and S. Strong, Science 261, 337 (1993).

${ }^{14}$ A similar situation happens in double-layered quantum Hall systems with spontaneous interlayer phase coherence: K. Yang et al., Phys. Rev. Lett. 72, 732 (1994); K. Moon et al., Phys. Rev. B 51, 5138 (1995); K. Yang et al., Phys. Rev. B 54, 11644 (1996).

15 S. Chakravarty, H.-Y. Kee and E. Abrahams, Phys. Rev. Lett. 82, 2366 (1999), and references therein.

(a)

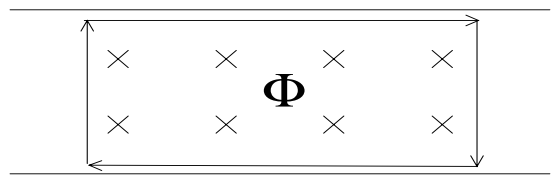

(b)

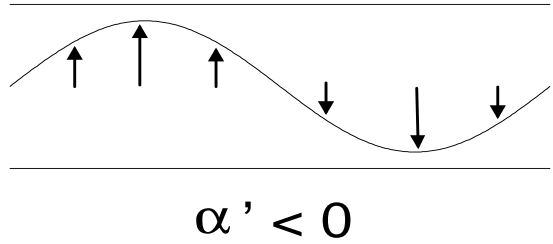

(c)

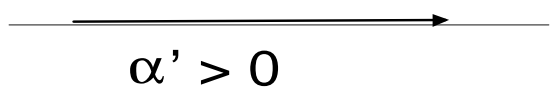

FIG. 1. (a) A closed path that encloses a finite amount of flux. (b) The current pattern in a bilayer system in the presence of strong parallel magnetic field, for in-plane mechanism of superconductivity. (c) Same as (b), for inter-layer pair hopping mechanism of superconductivity. 